\title{
Long-Range Spin-Qubit Interaction Mediated by Microcavity Polaritons
}

\author{
G. F. Quinteiro, ${ }^{1}$ J. Fernández-Rossier, ${ }^{2}$ and C. Piermarocchi ${ }^{1}$ \\ ${ }^{1}$ Department of Physics and Astronomy, Michigan State University, East Lansing, Michigan 48824, USA \\ ${ }^{2}$ Department of Applied Physics, University of Alicante, Alicante, 03690 Spain
}

(Received 28 March 2006; published 30 August 2006)

\begin{abstract}
We study the optically induced coupling between spins mediated by polaritons in a planar microcavity. In the strong-coupling regime, the vacuum Rabi splitting introduces anisotropies in the spin coupling. Moreover, due to their photonlike mass, polaritons provide an extremely long spin coupling range. This suggests the realization of two-qubit all-optical quantum operations within tens of picoseconds with spins localized as far as hundreds of nanometers apart.
\end{abstract}

DOI: 10.1103/PhysRevLett.97.097401

PACS numbers: 78.67. $-\mathrm{n}, 71.36 .+\mathrm{c}$

Planar microcavities are semiconductor devices that confine the electromagnetic field by means of two parallel semiconductor mirrors. When a quantum well (QW) is placed inside a planar microcavity, the optical excitations (excitons) in the QW couple to confined electromagnetic modes. In the strong-coupling regime, excitons and cavity photons give rise to new states, cavity polaritons [1,2], which appear in two branches separated by a vacuum Rabi splitting. In this Letter, we study the dynamics of localized spins in the QW interacting with cavity polaritons. Our calculations consider localized electron spins of shallow neutral donors in GaAs (e.g., $\mathrm{Si}$ ), but the theory is valid for other impurities and host semiconductors, as well as to charged quantum dots.

In the long wavelength limit, and for a cavity exactly at resonance with the exciton energy, polaritons can be seen as hybrid states that are exactly half matter and half light. However, even with a half-matter character, polaritons have photonlike dispersive properties, determined by the reduced mass of the photon-exciton system. A photon confined in a $\lambda / 2$ planar cavity has an effective mass of $M_{\gamma}=\hbar n \pi / c L$ ( $n$ is the refraction index and $L$ is the length of the cavity) which is typically 4 orders of magnitude smaller than the exciton mass. The small polariton mass is known to affect the dynamics of optical excitations with phonons [3,4], with interface disorder [5,6], and it suggests the possibility of polariton Bose-Einstein condensates at room temperature [7]. We will show below that the small polariton mass has also a strong effect on the mutual interaction of localized spins.

Proposals for quantum computers based on spin degrees of freedom require that individual qubits are placed close enough so to have a significant exchange interaction between them. This exchange interaction can be direct (i.e., induced by a controlled overlap of the wave functions), or indirect when mediated by spin excitations in a 2D electron gas [8] or by optical excitation across the semiconductor band gap [9-11]. In the indirect schemes, the range of the spin coupling is related to the mass of the mediating particles, and the coupling decreases exponentially as a function of the distance between the spins. Here, we show that the small polariton mass gives an extremely long range for the spin coupling. This implies that spin qubits can be located several hundreds of nanometers apart while still retaining control on pair interaction through the use of polaritons. We study two localized spins in a semiconductor quantum well embedded in a planar microcavity. The system is excited by an external monochromatic laser field with frequency below the lowest energy resonance. In response to the laser, the cavity becomes polarized, without energy absorption, and this dynamical polarization is used to control the localized spins.

In the following we derive the effective interactions between the two localized spins in the laser-driven planar microcavity. Because of spin-orbit coupling and quantum confinement we can restrict our discussion to the heavyhole valence band. Heavy holes have a total angular momentum component in the growth direction $j_{z}= \pm 3 / 2$. Combined with the electron in the conduction band, they give rise to either optically active excitons with $J_{z}= \pm 1$ or dark excitons with $J_{z}= \pm 2$ [we neglect the small LT splitting due to $e h$ exchange [12] ]. Under strong-coupling regime optically active excitons couple to cavity photons leading to upper and lower polaritons, whereas dark excitons remain unaffected. A nonperturbative description of the polaritons is accomplished by a Hopfield canonical transformation [13] that diagonalizes the interaction between $J_{z}= \pm 1$ excitons and cavity photons. The resulting dispersion of lower and upper polaritons reads

$$
\Omega_{k}^{\alpha}=\frac{\Omega_{k}^{X}+\Omega_{k}^{C}}{2}+\sigma_{\alpha} \sqrt{\frac{\left(\Omega_{k}^{X}-\Omega_{k}^{C}\right)^{2}}{4}+g_{k}^{2}},
$$

where $\alpha=\mathrm{LP}$ (UP) stands for lower (upper) polariton with $\sigma_{\mathrm{LP}}=-1(+1), \Omega_{k}^{X}=\epsilon_{0}+\hbar^{2} k^{2} / 2 M_{X}$ is the inplane dispersion of quantum well excitons, $\Omega_{k}^{C}=$ $\hbar c / n \sqrt{k^{2}+(\pi / L)^{2}}$ is the cavity mode dispersion, $g_{k}$ is the exciton-cavity-photon coupling. We show results for microcavities at resonance, i.e., satisfying the condition $\Omega_{0}^{C}=\Omega_{0}^{X}$, with a vacuum Rabi splitting of $2 g_{0}$. The Hamiltonian describing the free motion of the relevant quasiparticles in the system (lower polaritons with 
$J_{z}= \pm 1$, dark excitons with $J_{z}= \pm 2$, and upper polaritons with $J_{z}= \pm 1$ ) is denoted by $H_{0}$.

The spin of the electron in the excitons interacts, via a Heisenberg exchange, with the spin of the electrons localized in the impurities [11]. In the excitonic momentum representation the interaction reads

$$
H_{I}=\frac{J}{\mathcal{A}} \sum_{\vec{k}, \vec{k}^{\prime}, I} v_{k} v_{k^{\prime}} e^{i\left(\vec{k}-\vec{k}^{\prime}\right) \cdot \vec{R}_{I}} \vec{S}_{I} \cdot \vec{S}_{e},
$$

where the form factor $v_{k}=\left[1+(\Lambda k)^{2}\right]^{-1}$ [14] accounts for the short range of the exchange interaction and the finite size of the impurity state, $\mathcal{A}$ is the area of the cavity, $\vec{R}_{I}$ is the impurity location, and $\vec{S}_{e}$ and $\vec{S}_{I}$ are the excitonic and impurity spins, respectively. When represented in the polariton basis, this interaction acquires an additional form factor $r_{k}$ due to the fact that the (optically active) exciton is bound to a polariton. This interaction can turn a lower polariton into either a dark exciton or an upper polariton. Since we are considering microcavities in the strongcoupling regime excited below the lower polariton resonance, the interpolariton LP to UP conversion process is energy inhibited and we neglect it.

The microcavity is driven by $\sigma_{+}$circularly polarized laser field of frequency $\omega_{0}$ below the lower polariton branch and zero in-plane momentum. We define the detuning as $\delta=\Omega_{0}^{\mathrm{LP}}-\omega_{0}$. The laser-exciton Hamiltonian $H_{L}$ is characterized by the coupling constant $\mathcal{V}$. Only the excitonic component of the polariton at $k=0$ couples to the external laser field. We include the excitonic enhancement of the laser-matter coupling, proportional to the excitonic wave function $\phi_{1 s}=\sqrt{2 /\left(\pi a_{B}^{2}\right)}$. In the polariton representation, this coupling also acquires a Hopfield form factor $r_{0}=1 / \sqrt{2}$ [13]. At larger $k$ the Hopfield coefficient $r_{k}$ approaches one, since in that limit the lower polariton becomes excitonlike. The time dependent phase in $H_{L}$ is eliminated by transforming the Hamiltonian in the rotating frame at frequency $\omega_{0}$.

The effective Hamiltonian for the two localized spins is derived using the level shift operator $R\left(\omega_{0}\right)$ [15] to second order in the laser coupling $H_{L}$ :

$$
H_{\mathrm{eff}} \doteq \mathcal{P} R\left(\omega_{0}\right) \mathcal{P}=\mathcal{P} H_{L} \frac{\mathcal{Q}}{\omega_{0}-\mathcal{Q}\left(H_{0}+H_{I}\right) \mathcal{Q}} H_{L} \mathcal{P},
$$

where $Q$ is the projector operator onto the subspace of one excitation (polariton or dark exciton) plus the two spins, and $\mathcal{P}$ is the projector operator onto the subspace of the two spins without excitations. Equation (3) describes spinspin interactions to all orders in the exciton-impurity coupling. The two lowest order terms are obtained by expansion of the level shift operator in series of $H_{I}$. Therefore, our result is valid to second order both in the coupling with the external laser, $\mathcal{V}$, and in the exchange coupling $J$. However, since we are considering a microcavity in the strong-coupling regime, we keep all orders in the excitoncavity coupling by using the polariton picture.
The first order in the expansion of Eq. (3) in $H_{I}$ is an effective magnetic field proportional to the laser intensity. It describes the inverse Faraday effect of off-resonant circularly polarized radiation with frequency close to an optical resonance [16]. The inverse Faraday effect on impurities in semiconductors was investigated in Refs. [11,17], and was observed in Mn spins in quantum wells in Ref. [18]. This term disappears by using linearly polarized light because of time reversal symmetry.

The second order in $H_{I}$ plays the role of an effective interaction between the spins. We will focus our attention to this effective spin coupling, which is the one that can be used, for instance, to control spin entanglement and make optically controlled quantum gates. We eliminate the degrees of freedom of the itinerant particle by evaluating this term for the initial and final state of a polariton with wavevector $k=0$ and polarization $\sigma_{+}$. The final expression for the effective coupling reads

$$
H_{\mathrm{eff}}^{(2)}=J_{\mathrm{LP}}(R) s_{z}^{A} s_{z}^{B}+J_{X}(R)\left(s_{x}^{A} s_{x}^{B}+s_{y}^{A} s_{y}^{B}\right) .
$$

The relevant diagrams associated to these effective interactions are depicted in Fig. 1. They describe processes by which the laser excites a $k=0$ lower polariton that, after exchange interaction with one impurity, becomes either a dark exciton or a lower polariton with momentum $k$. After a second exchange interaction with another impurity the quasiparticle becomes a $k=0$ lower polariton that closes the diagram. Interestingly, the spin properties of the intermediate states are very different. If spin exchange occurs, the intermediate particle is a dark exciton whereas spin conserving exchange yields a lower polariton as an intermediate particle. The different dispersion of dark excitons and lower polaritons results in anisotropic interactions with different dependence on the interspin separation, $R$. In general $J_{\mathrm{LP}}(R) \neq J_{X}(R)$ so that (4) describes a spinanisotropic coupling. The two functions $J_{\mathrm{LP}}(R)$ and $J_{X}(R)$ describe the polariton-mediated and the excitonmediated contributions to the spin coupling and are defined as

$$
J_{\alpha}(R)=\frac{J^{2}|\mathcal{V}|^{2}\left|\phi_{1 s}\right|^{2}\left|r_{0}\right|^{2}}{\delta^{2}} \int_{0}^{\infty} \frac{d k}{2 \pi} \frac{\left(r_{k}^{2}\right)^{\nu_{\alpha}} v_{k}^{2} k J_{0}(k R)}{\left(\omega_{0}-\Omega_{k}^{\alpha}\right)},
$$

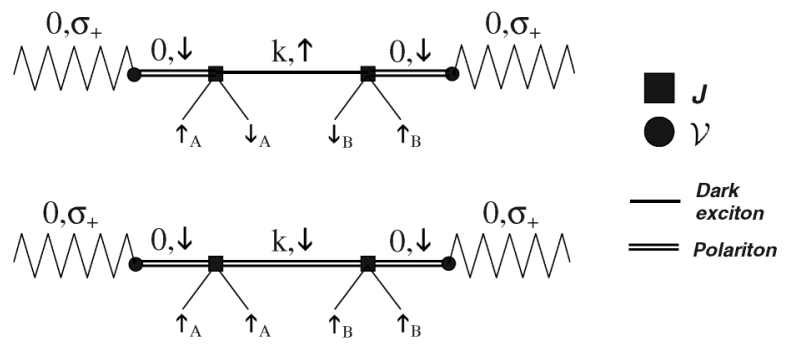

FIG. 1. Diagrams for the dark-exciton-mediated (upper) and lower polariton-mediated interactions between local spin $A$ and $B$. Zigzag lines represent the external laser, with 0 in-plane momentum. 
where $\alpha=\mathrm{LP}(X)$ denotes lower polariton (dark exciton) and $\nu_{\alpha}=1(0)$ for polaritons (dark excitons), $J_{0}$ is the Bessel function of order zero. Both $J_{\mathrm{LP}}$ and $J_{X}$ are proportional to the density of virtual polaritons $\left|r_{0}\right|^{2}|\mathcal{V}|^{2}\left|\phi_{1 s}\right|^{2} / \delta^{2}$. The cavity Rabi coupling splits dark and optically active excitations, making the indirect interaction mediated by polaritons and dark excitons different in strength and spin symmetry. The diagram with dark excitons as intermediate particles yields a transverse coupling whose intensity $J_{X}(R)$ is very similar to the optical RKKY interaction [9]. The polariton-mediated coupling $J_{\mathrm{LP}}(R)$ is spin conserving or Ising. Its strength differs from ORKKY due to the different dispersion and form factor for small momenta. In Fig. 2(a) we plot both $J_{X}(R)$ and $J_{\mathrm{LP}}(R)$ as a function of distance. The parameters chosen are characteristic of typical GaAs microcavity samples and are within the validity of our theoretical approach. We use excitonic atomic units where energy is given in excitonic $\mathrm{Ry}^{*}$ and lengths are in Bohr radius $a_{B}^{*}$. For GaAs, these units correspond to $1 \mathrm{Ry}^{*}=4.4 \mathrm{meV}$ and $a_{B}^{*} \sim 125 \AA$. It is apparent that at short distance $J_{X}$ and $J_{\mathrm{LP}}$ are comparable whereas at large distance $J_{X}$ vanishes and only the polariton-mediated interaction survives.

We now rewrite the effective coupling so that the extra effect of the cavity becomes evident. To do that we define $\delta J(R) \equiv J_{\mathrm{LP}}(R)-J_{X}(R)$, so that the effective coupling (4) reads:

$$
H_{\text {eff }}=J_{X}(R) \vec{s}^{A} \cdot \vec{s}^{B}+\delta J(R) s_{z}^{A} s_{z}^{B} .
$$

Although both the first and second terms have contributions from the two diagrams, their physical interpretation is clear: the first term is the standard optical exchange interaction and the second term represents the enhancement of the optical exchange interaction due to the presence of the cavity. In the limit of vanishing intracavity coupling, $g=$ 0 , we have $J_{\mathrm{LP}}=J_{X}$ so that $\delta J(R)=0$ and the effective interaction (6) becomes identical to the ORKKY. In Fig. 2(b) and 2(c) we plot both $J_{X}(R)$ and $\delta J(R)$ for the
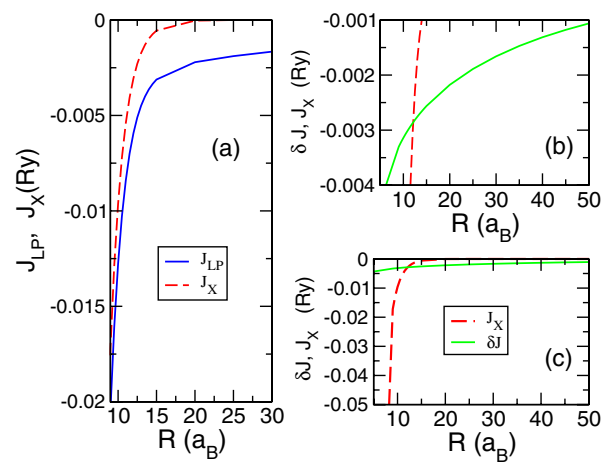

FIG. 2 (color online). Panel (a): optical exchange interactions mediate by excitons (dashed line) and by polaritons (solid lines). Panels (b), (c): cavity enhancement $\delta J$ and optical exchange interaction $J_{X}$ plotted in different vertical scales for the sake of clarity. In the plot we use $g_{0}=0.4 \mathrm{Ry}^{*}, \delta=0.15 \mathrm{Ry}^{*}, \mathcal{V}=$ $0.16 \mathrm{Ry}^{*}, \Lambda=0.25 a_{b}^{*}$, and $J=21 \mathrm{Ry}^{*}\left(a_{b}^{*}\right)^{2}$. same cavity of Fig. 2(a). It is apparent the extremely slow decay of $\delta J(R)$ as $R$ increases. In other words, the range of the optical 2-qubit interaction is dramatically increased by the presence of the cavity. The origin of this long-range coupling can be traced back to the fact that only small wave vectors contribute to $\delta J(R)$ : for a given cavity, there is a critical momentum $k_{c}$ above which the lower polariton is mostly a pure exciton. For $k>k_{c}$ both $r_{k} \sim 1$ and $\Omega_{k}^{P} \sim$ $\Omega_{k}^{X}$. The integrands of $J_{\mathrm{LP}}(R)$ and $J_{X}(R)$ coincide in that region. Therefore, the cavity enhancement comes exclusively from the small momentum region $k<k_{c}$.

In Fig. 3 we plot $J_{\mathrm{LP}}$ and $J_{X}$ in a logarithmic scale. For sufficiently large $R$, both $J_{X}$ and $J_{\mathrm{LP}}$ can be fitted to a Yukawa law: $J_{\alpha}(R) \simeq R^{-1 / 2} e^{-R / R_{0 \alpha}}$, where $\alpha=\mathrm{LP}$ or $X$ and $R_{0 \alpha}$ is the range of the corresponding interaction. As expected, $R_{0 \mathrm{LP}} \gg R_{0 X}$. In both cases the range of the interactions is related to the effective mass and the detuning of the corresponding quasiparticle, $R_{0 \alpha}^{-1}=\sqrt{2 M_{\alpha} \delta_{\alpha}}$. In the polaritonic case the value obtained from the fit matches $M_{\mathrm{LP}}$ defined from $\Omega_{k}^{\mathrm{LP}} \simeq \Omega_{0}^{\mathrm{LP}}+\frac{k^{2}}{2 M_{\mathrm{LP}}}$. For the values of Fig. 3 we have $M_{\mathrm{LP}}=10^{-5} m_{0}$, where $m_{0}$ is the free electron mass. Besides, for the excitonic case it can be analytically shown that $M_{\alpha=X}=M_{X}$ and $\delta_{X}=\delta+g_{0}$, which is the detuning of the dark exciton and the laser. Therefore, the long-range interaction of the cavityenhanced optical exchange interaction is related to the low effective mass of the lower polariton. The long-range nature of the polariton-mediated interaction presents important technological advantages for quantum information implementations. Using our parameters (see Fig. 1), we predict that the strength of the interaction is $\left|J_{\text {eff }}\right| \simeq$ $0.005 \mathrm{Ry}^{*}$ for impurities separated by distances of the order of $R_{c}=12 a_{b}^{*} \simeq 150 \mathrm{~nm}$. An estimate for the time needed for an operation can be given as $T=\pi /\left(2\left|J_{\text {eff }}\right|\right) \simeq$ $40 \mathrm{ps}$, which is much smaller than the typical dephasing time for impurity spin qubits. Recent measurements have reported a spin relaxation time of the order of $\mu$ s for donors in GaAs [19]. We expect the spin decoherence time $\left(T_{2}\right)$ of a single donor in GaAs to be equal or larger than the observed [20] $T_{2} \simeq 10 \mathrm{~ns}$ in a quantum dot, caused by the coupling to the nuclei. Moreover, notice that the time needed for a quantum operation does not change consid-

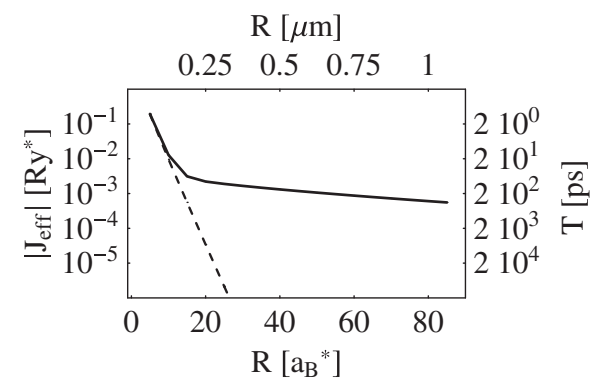

FIG. 3. Logarithmic plot of $J_{X}$ (dashed line) and $J_{\mathrm{LP}}$ (solid line), that shows their different range and $\left(g_{0}=0.4 \mathrm{Ry}^{*}\right.$, other parameters as in Fig. 2). 
erably when we further increase the qubit separation. Even with an interqubit separation of $1 \mu \mathrm{m}$ the time needed for one operation increases only by 1 order of magnitude to about $400 \mathrm{ps}$, and is still reasonably smaller than the decoherence time. In principle the finite lifetime of polaritons (typically of the order of $1 \mathrm{~ns}$ ) may affect the fidelity of the two-qubit operation. This was recently investigated in a similar system in Ref. [21]. This study showed that errors are small (below the threshold for quantum error correction) when the detuning is large compared to the linewidth and the operations are carried out adiabatically. The long-range interaction simplifies the realization of electric gates to control one-qubit operations and the use of localized magnetic field becomes feasible. These onequbit operations combined with the Ising interaction permit to implement the 2-qubit CNOT operation [22].

The polariton-mediated coupling could be also used to control the nuclear spin of the donor in a scheme similar to the one in Ref. [8]. In contrast to other cavity QED-based quantum computing implementations [23], the scheme discussed here does not require 0D confined electromagnetic modes, which is much harder to achieve experimentally. In a planar cavity the lateral dimension is not limited by the optical wavelength, which provides a fully scalable geometry for the qubits.

A spin coupling can also be obtained by a real polariton population in a scheme analogue to the standard RKKY [24] spin coupling. The spin interaction induced by a OD cavity and excitons in quantum dots has been recently investigated [25], and also in this 0D case the presence of a strong coupling generates anisotropies in the spin interaction. The use of real carriers is not appealing for quantum computing implementation since it adds decoherence to the spin-qubit. However, it would be interesting to explore the dynamics of spin in the presence of a dense polaritons population that condense in a phase coherent state, as observed recently in II-VI microcavities [26]. High-quality microcavities embedding Mn-doped magnetic quantum wells in the strong-coupling regime have recently been realized [27]. The polariton-mediated spin coupling could be explored in these systems as a method for the ultrafast control of the quantum well magnetization [28].

In conclusion, we have shown that the optical excitation of microcavity polaritons can couple spins localized in a quantum well. Because of the small polariton mass, the spin coupling has an extremely long range, and at large distances is Ising-like. The interaction is strong enough for the realization of quantum operations with spins located as far as several hundred of nanometers apart and within a time scale much shorter than the spin decoherence time. This interplay of polaritons and localized spins represents a peculiar feature of solid-state cavity QED, which has no equivalent in the atomic case.

This work was supported by the National Science Foundation No. DMR-0605801, the Donald D. Harrington Fellows Program at The University of Texas at Austin, the Spanish Ministry of Education and Generalitat Valenciana for Grants No. FIS200402356 and No. GV05152 as well as Ramon y Cajal Program.

[1] C. Weisbuch, M. Nishioka, A. Ishikawa, and Y. Arakawa, Phys. Rev. Lett. 69, 3314 (1992).

[2] V. Savona, C. Piermarocchi, A. Quattropani, P. Schwendimann, and F. Tassone, Phase Transit. 68, 169 (1999).

[3] G. Cassabois et al., Phys. Rev. B 61, 1696 (2000).

[4] V. Savona and C. Piermarocchi, Phys. Status Solidi A 164, 45 (1997).

[5] D. M. Whittaker et al., Phys. Rev. Lett. 77, 4792 (1996).

[6] V. Savona, C. Piermarocchi, A. Quattropani, F. Tassone, and P. Schwendimann, Phys. Rev. Lett. 78, 4470 (1997).

[7] H. Deng, G. Weihs, C. Santori, J. Bloch, and Y. Yamamoto, Science 298, 199 (2002).

[8] D. Mozyrsky, V. Privman, and M. L. Glasser, Phys. Rev. Lett. 86, 5112 (2001).

[9] C. Piermarocchi, Pochung Chen, L. J. Sham, and D. G. Steel, Phys. Rev. Lett. 89, 167402 (2002).

[10] G. Ramon, Y. Lyanda-Geller, T. L. Reinecke, and L. J. Sham, Phys. Rev. B 71, 121305(R) (2005).

[11] C. Piermarocchi and G.F. Quinteiro, Phys. Rev. B 70, 235210 (2004).

[12] O. Ikeuchi, S. Adachi, H. Sasakura, and S. Muto, J. Appl. Phys. 93, 9634 (2003).

[13] J. J. Hopfield, Phys. Rev. 112, 1555 (1958).

[14] Y. Yamaguchi, Phys. Rev. 95, 1628 (1954).

[15] C. Cohen-Tannoudji, J. Dupont-Roc, and G. Grynberg, Atom-Photons Interactions (Wiley, New York, 1998), Chap. III.

[16] L. D. Landau and E. M. Lifshitz, Electrodynamics of Continuous Media (Pergamon, New York, 1984), Chap. XI.

[17] M. Combescot and O. Betbeder-Matibet, Solid State Commun. 132, 129 (2004).

[18] A. Gupta, R. Knobel, N. Samarth, and D. D. Awschalom, Science 292, 2458 (2001).

[19] Kai-Mei C. Fu, C. Santori, C. Stanley, M. C. Holland, and Yoshihisa Yamamoto, Phys. Rev. Lett. 95, 187405 (2005).

[20] J. R. Petta et al., Science 309, 2180 (2005).

[21] G.F. Quinteiro and C. Piermarocchi, Phys. Rev. B 72, 045334 (2005).

[22] N. A. Gershenfeld and I. L. Chuang, Science 275, 350 (1997).

[23] A. Imamoglu et al., Phys. Rev. Lett. 83, 4204 (1999).

[24] M. A. Ruderman and C. Kittel, Phys. Rev. 96, 99 (1954); T. Kasuya, Prog. Theor. Phys. 16, 45 (1956); K. Yosida, Phys. Rev. 106, 893 (1957).

[25] G. Chiappe, J. Fernández-Rossier, E. Louis, and E. V. Anda, Phys. Rev. B 72, 245311 (2005).

[26] M. Richard, J. Kasprzak, R. Romestain, R. André, and Le Si Dang, Phys. Rev. Lett. 94, 187401 (2005).

[27] S. Bahbah, R. Frey, R. André, G. Roosen, and C. Flytzanis, Solid State Commun. 136, 147 (2005).

[28] J. Fernández-Rossier, C. Piermarocchi, P. Chen, A. H. MacDonald, and L. J. Sham, Phys. Rev. Lett. 93, 127201 (2004). 